\title{
Suppression of fibroblast growth factor-2 expression: Possible mechanism underlying methylmercury-induced inhibition of the repair of wounded monolayers of cultured human brain microvascular endothelial cells
}

\author{
Takashi Hirooka1, Yasuyuki Fujiwara², Shoko Inoue³, Yasuhiro Shinkai1, \\ Chika Yamamoto ${ }^{1,3}$, Masahiko Satoh ${ }^{2}$, Akira Yasutake ${ }^{4}$, Komyo Eto ${ }^{5}$ and Toshiyuki Kaji1,3 \\ ${ }^{\prime}$ Organization of Frontier Research for Preventive Pharmaceutical Science, Hokuriku University, Ho-3 \\ Kanagawa-machi, Kanazawa 920-1181, Japan \\ ${ }^{2}$ Laboratory of Pharmaceutical Health Sciences, School of Pharmacy, Aichi Gakuin University, 1-100 Kusumoto-cho, \\ Chikusa-ku, Nagoya, Aichi 464-8650, Japan \\ ${ }^{3}$ Department of Environmental Health, Faculty of Pharmaceutical Sciences, Hokuriku University, Ho-3 \\ Kanagawa-machi, Kanazawa 920-1181, Japan \\ ${ }^{4}$ National Institute for Minamata Disease, 4058-18 Hama, Minamata 867-0008, Japan \\ ${ }^{5}$ Health and Nursing Facilities for the Aged, Jushindai, Shinwakai, 272 Ikura Kitakawa, Tamana 865-0041, Japan
}

(Received April 30, 2009; Accepted May 13, 2009)

\begin{abstract}
Vascular toxicity is an important feature of the neuropathy induced by methylmercury. Methylmercury does not cause nonspecific cell damage, but rather retards the repair of wounded monolayers of cultured human brain microvascular endothelial cells by inhibiting their proliferation. Since vascular endothelial cell proliferation during the repair process strongly depends on the fibroblast growth factor-2 (FGF-2) system, we investigated the effects of methylmercury on the expression of FGF-2 and related proteins (i.e., FGF receptor 1 and perlecan) in cultured human brain microvascular endothelial cells. Of the mRNAs examined, FGF-2 mRNA expression was significantly lowered by methylmercury in not only wounded monolayers but also dense and sparse cultures of endothelial cells; a lower expression of FGF-2 protein in the cells was confirmed. In addition, exogenous FGF-2 partially abrogated the proliferation-inhibitory effect of methylmercury. The results of this study suggest that suppression of FGF2 expression is one of the mechanisms underlying the inhibitory effect of methylmercury in damaged endothelial cell monolayers. The FGF-2 system may be one of the important biological systems behind the vascular toxicity of methylmercury.
\end{abstract}

Key words: Methylmercury, Endothelial cell, Fibroblast growth factor 2, Microvascular

\section{INTRODUCTION}

Methylmercury is a potent neurotoxicant that causes severe neuropathy in humans and animals (Sanfeliu et al., 2003), as is observed in patients with Minamata disease. However, the pathogenesis of methylmercury poisoning is not yet completely understood. Neuropathological changes are generally restricted to specific areas of the brain: the granular layers of the cerebellum and the calcarine, postcentral, precentral, and temporal transverse regions of the cerebral cortex in patients with Minamata disease
(Eto, 1997). An experimental study suggested that the neuronal damage induced by methylmercury is a secondary manifestation of the edematous changes in the white matter around the deep sulci (Eto et al., 2001). Since brain edema is defined as an abnormal accumulation of body fluid within brain parenchyma due to the disruption of the blood-brain barrier or fluid accumulation in injured cells (Iencean, 2003; Marmarou, 2004), we hypothesize that the types of cells constituting the brain microvessels (i.e., endothelial cells and pericytes) that lie around neurons may be the targets for methylmercury toxicity.

Correspondence: Toshiyuki Kaji (E-mail: t-kaji@hokuriku-u.ac.jp) 
Endothelial cells form a monolayer that covers the inner surface of brain microvessels and pericytes are located around and along the endothelial cells. Endothelial cells form tight junctions and regulate the selective transport of ions and nutrients such as amino acids and glucose, and thus function as blood-brain barriers (Abbott et al., 2006). Most of the previous studies on methylmercury neurotoxicity have focused on cells other than vascular cells, including astrocytes and neurons. However, it is important to elucidate the functional damage caused to endothelial cells and pericytes if we are to understand the causes of brain edema in such cases. We have previously reported that methylmercury does not cause nonspecific cell damage but instead retards the repair of wounded monolayers of cultured human brain microvascular endothelial cells by inhibiting their proliferation (Hirooka et al., 2007). However, the biological system(s) responsible for this inhibitory effect has/have not yet been identified.

The regulation of vascular endothelial cell functions such as proliferation, migration, fibrinolysis, and extracellular matrix formation strongly depends on endogenous growth factors/cytokines, particularly fibroblast growth factor-2 (FGF-2) (Sato and Rifkin, 1988). It has been shown that the FGF-2 system can be a target for heavy-metal toxicity. For example, lead, like methylmercury, inhibits the repair of wounded monolayers of vascular endothelial cells (Fujiwara et al., 1997) by inhibiting their proliferation (Kaji et al., 1995). The inhibitory effect of lead on the proliferation of vascular endothelial cells is attributed to a decreased ability to respond to endogenous FGF-2 (Fujiwara and Kaji, 1999a). This occurs via the inhibition of the synthesis of perlecan (Fujiwara and Kaji, $1999 b)$, which is a large heparan sulfate proteoglycan that promotes the binding of FGF-2 to its receptor (Aviezer et al., 1994a).

The present study was undertaken to investigate the effects of methylmercury on the expression of FGF-2 and related proteins, i.e., FGF receptor 1 (FGFR-1) and perlecan, in cultured human brain microvascular endothelial cells. We observed that the suppression of FGF-2 expression was the possible mechanism underlying the methylmercury-induced inhibition of vascular endothelial cell proliferation.

\section{MATERIALS AND METHODS}

\section{Materials}

Human brain microvascular endothelial cells were purchased from DS Pharma Biomedical (Osaka, Japan). The following materials were purchased from the respective vendors: HuMedia EG-2 (a growth medium for human endothelial cells), Kurabo (Osaka, Japan); Dulbecco's modified Eagle's medium (DMEM) and calcium- and magnesium-free phosphate-buffered saline (CMF-PBS), Nissui Pharmaceutical (Tokyo, Japan); fetal bovine serum (FBS), Invitrogen (Carlsbad, CA, USA); bovine serum albumin (BSA), Sigma-Aldrich Chemical (St. Louis, MO, USA); collagen-coated tissue culture dishes and plates, AGC Techno Glass (Chiba, Japan); methylmercury chloride, Tokyo Chemical Industry (Tokyo, Japan); the HighCapacity cDNA Reverse Transcription Kit, TaqMan Gene Expression Master Mix, and TaqMan primer and probe pairs for real-time reverse-transcription polymerase chain reaction (RT-PCR), Applied Biosystems (Foster, CA, USA); rabbit monoclonal anti-FGF-2 antibody (19A9) and horseradish peroxidase conjugated anti-rabbit $\mathrm{IgG}$ antibody, Cell Signaling (Beverly, MA, USA); enhanced chemiluminescence (ECL) Western blotting detection reagents, Nacalai Tesque (Kyoto, Japan); polyvinylidene difluoride (PVDF) membrane, Millipore (Billerica, MA, USA); and L-cysteine and other reagents, Nacalai Tesque (Kyoto, Japan).

\section{Cell culture}

Human brain microvascular endothelial cells were cultured at $37^{\circ} \mathrm{C}$ until confluence in HuMedia EG-2 in 100 $\mathrm{mm}$ dishes in a humid atmosphere with $5 \% \mathrm{CO}_{2}$. The cells were then transferred to other $100-\mathrm{mm}$ dishes or sixwell culture plates at a density of $5 \times 10^{3}$ cells $/ \mathrm{cm}^{2}$ and cultured for $24 \mathrm{hr}$ in HuMedia EG-2 (sparse culture) or until confluence (dense culture). In another experiment, the monolayers of dense cultures were wounded with a cell scraper, and the damaged monolayer was washed twice with fresh HuMedia EG-2 to remove detached cells (wounded culture).

\section{Repair assay}

Wounded cultures of human brain microvascular endothelial cells were treated with methylmercury $(1,2$, and $3 \mu \mathrm{M}$ ) in the absence or presence of $15 \mathrm{ng} / \mathrm{ml} \mathrm{FGF-}$ 2 for $24 \mathrm{hr}$ at $37^{\circ} \mathrm{C}$ in $1 \mathrm{ml}$ of fresh HuMedia EG-2 in six-well culture plates. After treatment, the medium was discarded and the cells washed with CMF-PBS. The cells were fixed in methanol, stained with Giemsa, and morphologically examined.

\section{Real-time RT-PCR}

Wounded, dense, and sparse cultures of human brain microvascular endothelial cells were each treated with methylmercury $(1,2$, and $3 \mu \mathrm{M})$ at $37^{\circ} \mathrm{C}$ for $24 \mathrm{hr}$ in HuMedia EG-2. Subsequently, the conditioned medi- 
The FGF-2 system and the vascular toxicity of methylmercury

um was discarded and the cells were washed twice with CMF-PBS. The total RNA was extracted using the RNeasy Lipid Tissue Mini Kit (Qiagen, Tokyo, Japan). The cDNA was synthesized from the mRNA using the HighCapacity cDNA Reverse Transcription Kit. Real-time RTPCR was performed in triplicate using the TaqMan Gene Expression Assay on a 7500 real-time PCR system and the expression of mRNAs was analyzed by the comparative $C t$ method using glyceraldehydes-3-phosphate dehydrogenase as an endogenous control. The primer and probe sets for FGF-2 (Hs 00266645 ml), FGFR1 (Hs $00241111 \mathrm{ml}$ ), and the perlecan core protein (Hs 00194179_ml) were selected from the TaqMan Gene Expression Assay (Applied Biosystems).

\section{Western blot analysis of FGF-2}

Dense cultures of human brain microvascular endothelial cells were treated with methylmercury $(1,2$, and 3 $\mu \mathrm{M})$ at $37^{\circ} \mathrm{C}$ for $24 \mathrm{hr}$ in HuMedia EG-2. After treatment, the conditioned medium was discarded and the cell layer washed twice with ice-cold CMF-PBS containing $2 \mathrm{mM}$ ethylene glycol tetraacetic acid (EGTA). Cell lysates were prepared by adding $50 \mathrm{mM}$ Tris- $\mathrm{HCl}$ buffer ( $\mathrm{pH}$ 6.8) containing 10\% glycerol and 2\% SDS to the cell layers and incubating them at $95^{\circ} \mathrm{C}$ for $5 \mathrm{~min}$. The protein $(40 \mu \mathrm{g})$ was separated by $12 \%$ SDS-polyacrylamide gel electrophoresis and transferred onto a PVDF membrane. The membrane was blocked for $1 \mathrm{hr}$ in $20 \mathrm{mM}$ Tris- $\mathrm{HCl}$ buffer containing 5\% skin milk, $150 \mathrm{mM} \mathrm{NaCl}$, and $0.1 \%$ Tween 20 ( $\mathrm{pH} 7.5$ ) before being incubated with a rabbit monoclonal antibody against FGF-2 (1:200) for $1 \mathrm{hr}$ at room temperature. The blot was probed with horseradish peroxidase-conjugated anti-rabbit IgG and the bands were visualized using the ECL procedure. The chemiluminescent intensity of the bands was quantified using Image-J software. The intensity of the FGF-2 band was normalized by that of the corresponding actin band.

\section{Statistical analysis}

The statistical significance of the data was determined using analysis of variance and Bonferroni's multiple $t$ test. $P<0.05$ was considered statistically significant.

\section{RESULTS}

Fig. 1 shows the morphological features of the wounded monolayers of human brain microvascular endothelial cells after $24 \mathrm{hr}$ of exposure to methylmercury. Generally, after wound induction, the damaged monolayer is repaired by cell migration and proliferation. Methylmercury inhibited the repair of the damaged monolayer in a concentration-dependent manner without any cell damage.

Fig. 2 shows the effects of methylmercury on the expression of mRNAs encoding FGF-2 and related proteins (i.e., FGFR-1 and the perlecan core protein) in wounded endothelial monolayers, dense cultures (mitotically inactive), and sparse cultures (mitotically active). We observed that methylmercury strongly suppressed the expression of FGF-2 mRNA in a concentration-dependent manner in the wounded monolayers, dense cultures, and sparse cultures of the endothelial cells. Furthermore, methylmercury moderately reduced the expression of FGFR-1 mRNA and did not affect perlecan mRNA expression. We failed to detect the expression of FGFR2, FGFR-3, and FGFR-4 mRNAs. In fact, methylmercury caused a significant reduction in the expression of FGF-2 in the dense cultures (Fig. 3).

To examine whether the administration of exogenous FGF-2 could restore the repair process in the wounded endothelial cell monolayers, the wounded monolayers were incubated with methylmercury in the presence of

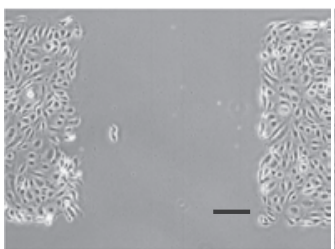

Before exposure

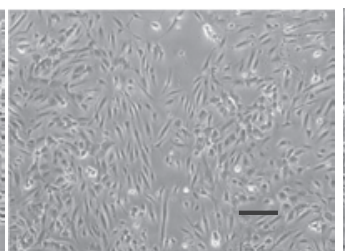

Control

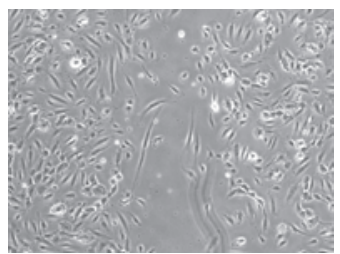

1

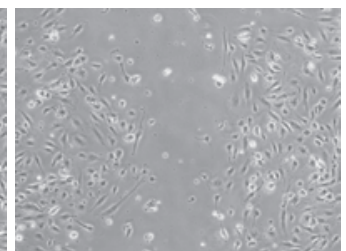

2

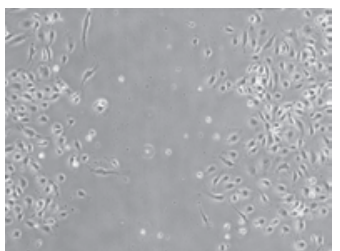

3

Methylmercury $(\mu \mathrm{M})$

Fig. 1. Morphology of the wounded monolayers of human brain microvascular endothelial cells after exposure to methylmercury. Dense cultures of human brain microvascular endothelial cells were wounded and then incubated at $37^{\circ} \mathrm{C}$ for $24 \mathrm{hr}$ in the presence of methylmercury $(1,2$, and $3 \mu \mathrm{M})$. Phase-contrast microscopy images were obtained before and after treatment with methylmercury $(\times 40)$. Scale bar $=250 \mu \mathrm{m}$. 

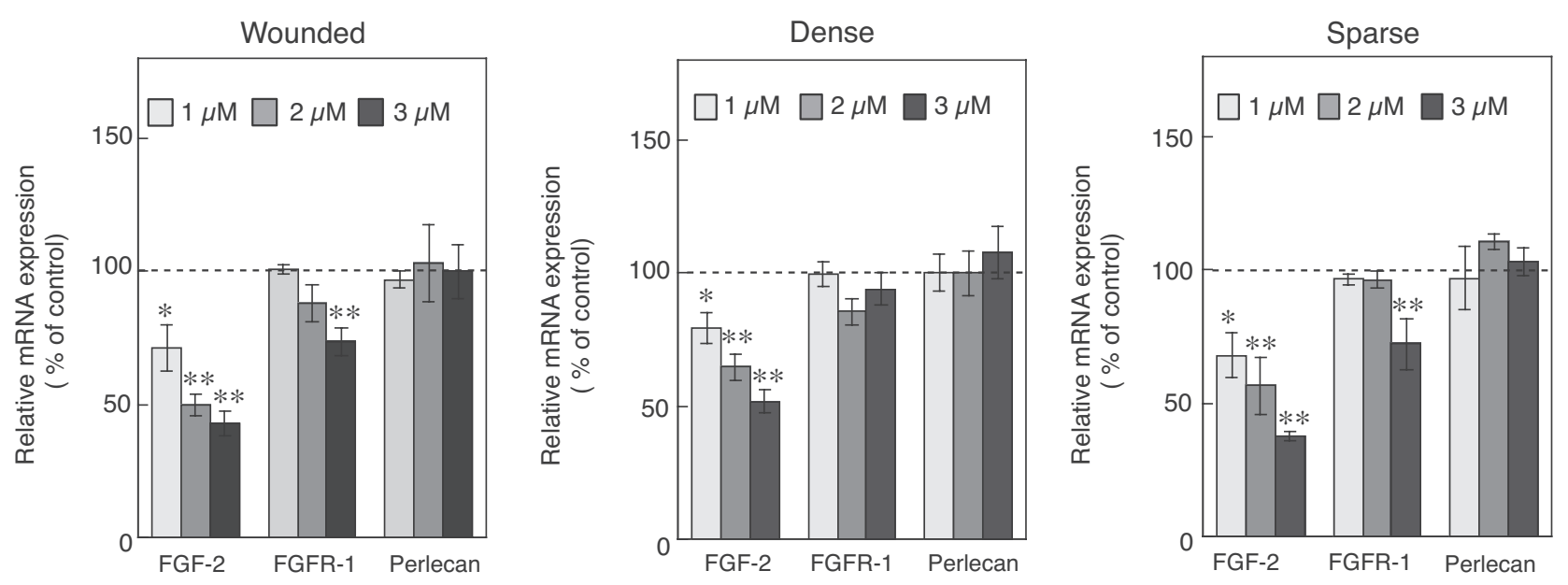

Fig. 2. The expression of the mRNAs encoding FGF-2, FGFR-1, and the perlecan core protein in human brain microvascular endothelial cells after exposure to methylmercury. Wounded monolayers, dense cultures, and sparse cultures of the cells were incubated at $37^{\circ} \mathrm{C}$ for $24 \mathrm{hr}$ in the presence of methylmercury $(1,2$, and $3 \mu \mathrm{M})$. Error bars represent the standard error $(\mathrm{n}=$ 3). Significantly different from the corresponding control, $* P<0.05$; ** $P<0.01$.

$[\mathrm{A}]$

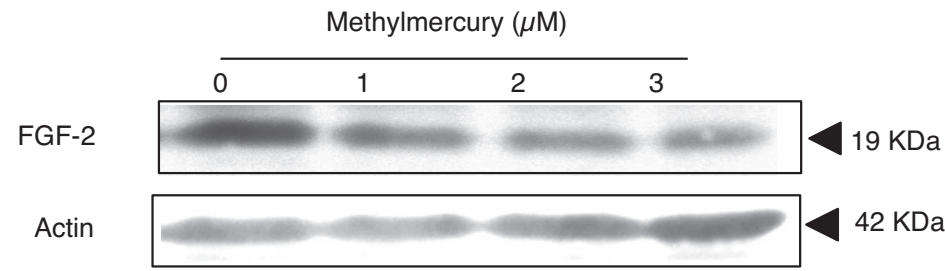

[B]

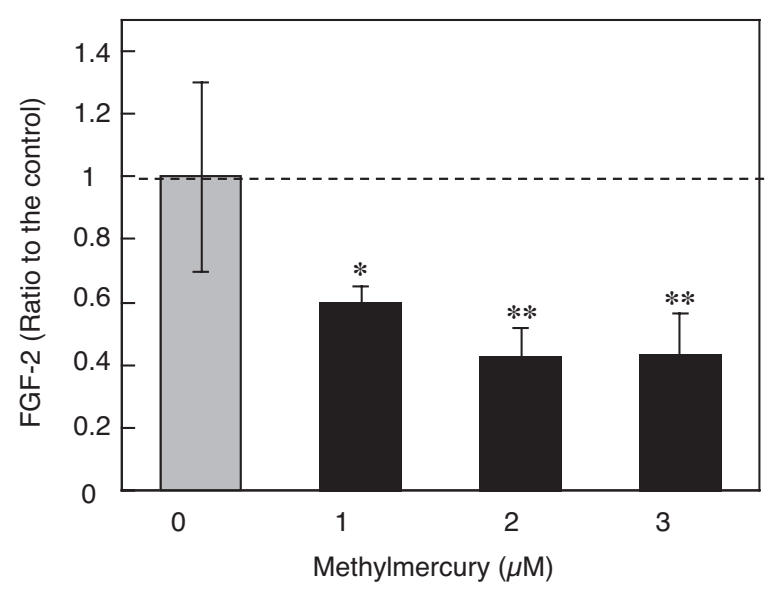

Fig. 3. Western blot analysis of FGF-2 in human brain microvascular endothelial cells after exposure to methylmercury. Dense cultures of the cells were incubated at $37^{\circ} \mathrm{C}$ for $24 \mathrm{hr}$ in the presence of methylmercury $(1,2$, and $3 \mu \mathrm{M})$. [A] A representative fluorograph. [B] The relative intensity of the bands is represented as the ratio to the mean of the control $(\mathrm{n}=4)$. Error bars represent the standard error. Significantly different from the control, $* P<0.05 ; * * P<0.01$.

exogenous FGF-2 and the repair of the wounded areas of the monolayer was observed morphologically. As shown in Fig. 4, methylmercury inhibited the repair of monol- ayers in a concentration-dependent manner, and the inhibition was partly prevented in the presence of exogenous FGF-2. 
The FGF-2 system and the vascular toxicity of methylmercury

$[A]$

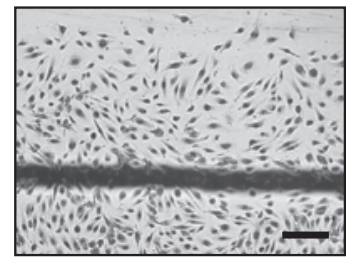

Control

[B]

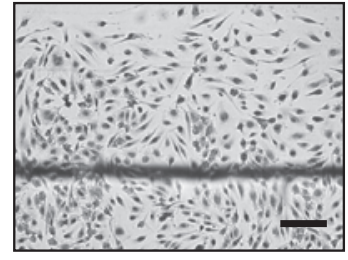

Control

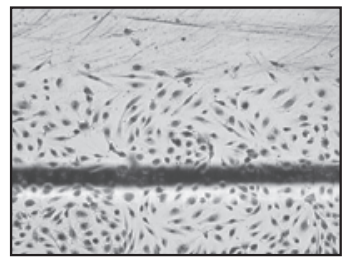

1

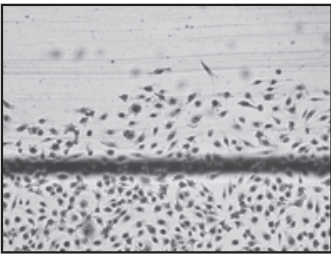

2

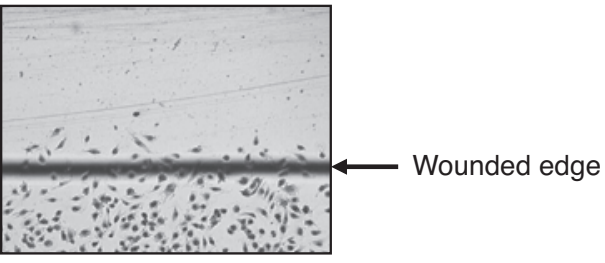

3

Methylmercury $(\mu \mathrm{M})$

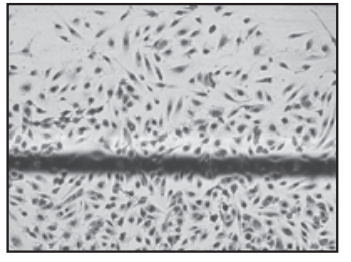

1

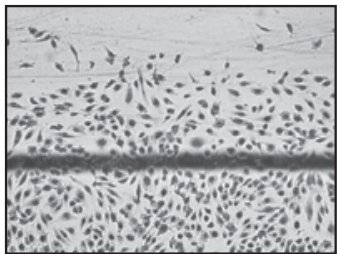

2

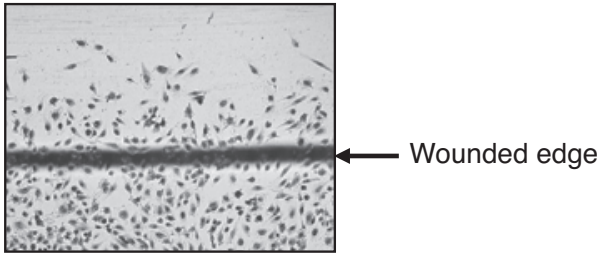

3

Methylmercury $(\mu \mathrm{M})$

Fig. 4. Morphology of the wounded monolayers of human brain microvascular endothelial cells after exposure to methylmercury in $[\mathrm{A}]$ the absence or [B] the presence of exogenous FGF-2. Dense cultures of human brain microvascular endothelial cells were wounded and then incubated at $37^{\circ} \mathrm{C}$ for $24 \mathrm{hr}$ with methylmercury $(1,2$, and $3 \mu \mathrm{M})$ in the absence or presence of human recombinant FGF-2 $(15 \mathrm{ng} / \mathrm{ml})$. Giemsa-stained images $(\times 40)$. Scale bar $=250 \mu \mathrm{m}$.

\section{DISCUSSION}

Abnormal maturation and barrier dysfunction of endothelial microvessels are the most important events in the pathogenesis of brain disorders caused by heavy metal toxicity (Zheng et al., 2003). Although it has been suggested that microvessels in the brain are one of the targets of methylmercury (Eto, 1997; Bertossi et al., 2004), the vascular toxicity of methylmercury is still unclear. The results of this study indicate that the suppression of FGF-2 expression is one of the mechanisms underlying the inhibitory effect of methylmercury on endothelial cell proliferation (Hirooka et al., 2007), thereby resulting in the retardation of the repair of the wounded monolayers. It has been shown that the estimated threshold blood concentration of mercury is estimated to be approximately 1 $\mu \mathrm{M}$ for the onset of intoxication and 1-5 $\mu \mathrm{M}$ for pathological changes such as paresthesia, ataxia, and visual changes (Bakir et al., 1973; Sanfeliu et al., 2003). These concentrations are consistent with those used in this study, supporting the hypothesis that the present data may possibly reflect the cellular events caused by methylmercury in vivo.

The FGF-2 system has been postulated to stimulate vascular endothelial cells in an autocrine fashion only after leakage of FGF-2 from severely damaged endothelial cells (Bikfalvi et al., 1997) because there is no signal sequence in FGF-2 that directs its release via the normal secretory pathway (Rifkin and Moscatelli, 1989). It should also be noted that heparan sulfate chains markedly promote the binding of FGF-2 to FGFR (Yayon et al., 1991). Endothelial heparan sulfate chains bind to FGF-2 and stabilize the growth factor by protecting it from degradation by proteinases (Saksela et al., 1988). The formation of FGF-2-heparan sulfate complexes enhances the levels of FGF-2 expression at cell surfaces and enables long-term stimulation of endothelial cell functions such as proliferation (Flaumenhaft et al., 1989). Heparan sulfate chains of perlecan-a large heparan sulfate proteoglycanpromote the binding of FGF-2 to FGFR, whereas heparan sulfate chains of other proteoglycan species compete for this receptor (Aviezer et al., 1994a, 1994b; Knox et al., 2002). Indeed, perlecan is the predominant heparan sulfate proteoglycan in human brain microvascular endothelial cells (Yamamoto et al., 2005). In the present study, we observed that methylmercury concentration-dependently reduced the expression of FGF-2, moderately reduced the expression of FGFR-1, and did not affect the expression of perlecan, suggesting that FGF-2 is one of the molecular targets of methylmercury. In fact, the presence of 
exogenous FGF-2 could partially abrogate the inhibitory effect of methylmercury, supporting the hypothesis that the FGF-2 system is the primary target of methylmercury toxicity in human brain microvascular endothelial cells.

In conclusion, our results indicate that suppression of FGF-2 expression is a possible mechanism underlying the methylmercury-induced inhibition of wounded monolayers of cultured human brain microvascular endothelial cells. In other words, the FGF-2 system is the major target of methylmercury toxicity. The present study also shows that the FGF-2 system is a target for methylmercury and lead (Fujiwara and Kaji, 2000). However, it should be noted that in lead toxicity, perlecan in the FGF-2 system is the target molecule (Fujiwara and Kaji, 1999b), whereas in methylmercury poisoning, FGF-2 itself is the target molecule.

\section{ACKNOWLEDGMENTS}

This work was supported by the "Academic Frontier" Project for Private Universities from the Ministry of Education, Culture, Sports, Science, and Technology of Japan, 2005-2009; a Grant-in-Aid for Young Scientists (B) from the Ministry of Education, Culture, Sports, Science, and Technology of Japan (to T.H.); a Grant-in-Aid for Young Scientists (B) from the Ministry of Education, Culture, Sports, Science, and Technology of Japan (to Y.F.); a Grant-in-Aid for Scientific Research (C) from the Japan Society for the Promotion of Science (to C.Y.); and a Grant-in-Aid for Scientific Research (B) from the Japan Society for the Promotion of Science (to T.K.).

\section{REFERENCES}

Abbott, J.N., Rönnbäck, L. and Hansson, E. (2006): Astrocyteendothelial interactions at the blood-brain barrier. Nat. Rev. Neurosci., 7, 41-53.

Aviezer, D., Hecht, D., Safran, M., Eisinger, M., David, G. and Yayon, A. (1994a): Perlecan, basal lamina proteoglycan, promotes basic fibroblast growth factor-receptor binding, mitogenesis, and angiogenesis. Cell, 79, 1005-1013.

Aviezer, D., Levy, E., Safran, M., Svahn, C., Buddecke, E., Schmidt, A., David, G., Vlodavsky, I. and Yayon, A. (1994b): Differential structural requirements of heparin and heparan sulfate proteoglycans that promote binding of basic fibroblast growth factor to its receptor. J. Biol. Chem., 269, 114-121.

Bakir, F., Damluji, S.F., Amin-Zaki, L., Murtadha, M., Khalidi, A., Al-Rawi, N.Y., Tikriti, S., Dhahir, H.I., Clarkson, T.W., Smith, J.C. and Doherty, R.A. (1973): Methylmercury poisoning in Iraq: An interuniversity report. Science, 181, 230-241.

Bertossi, M., Girolamo, F., Errede, M., Virgintino, D., Elia, G., Ambrosi, L. and Roncali, L. (2004): Effects of methylmercury on the microvasculature of the developing brain. Neurotoxicology, $\mathbf{2 5}, 849-857$.

Bikfalvi, A., Klein, S., Pintucci, G. and Rifkin, D.B. (1997): Biolog- ical roles of fibroblast growth factor-2. Endocr. Rev., 18, 26-45.

Eto, K. (1997): Pathology of Minamata disease. Toxicol. Pathol., 25, 614-623.

Eto, K., Yasutake, A., Kuwana, T., Korogi, Y., Akima, M., Shimozeki, T., Tokunaga, H. and Kaneko, Y. (2001): Methylmercury poisoning in common marmosets-a study of selective vulnerability within the cerebral cortex. Toxicol. Pathol., 29, 565-573.

Flaumenhaft, R., Moscatelli, D., Saksela, O. and Rifkin, D.B. (1989): Role of extracellular matrix in the action of basic fibroblast growth factor: matrix as a source of growth factor for longterm stimulation of plasminogen activator production and DNA synthesis. J. Cell. Physiol., 140, 75-81.

Fujiwara, Y. and Kaji, T. (1999a): Possible mechanism for lead inhibition of vascular endothelial cell proliferation: a lower response to basic fibroblast growth factor through inhibition of heparan sulfate synthesis. Toxicology, 133, 147-157.

Fujiwara, Y. and Kaji, T. (1999b): Lead inhibits the core protein synthesis of a large heparan sulfate proteoglycan perlecan by proliferating vascular endothelial cells in culture. Toxicology, 133, 159-169.

Fujiwara, Y. and Kaji, T. (2000): Inhibition of the repair of injured endothelial cell monolayers by lead and its possible mechanisms. J. Health Sci., 46, 1-4.

Fujiwara, Y., Kaji, T., Sakurai, S., Sakamoto, M. and Kozuka, H. (1997): Inhibitory effect of lead on the repair of wounded monolayers of cultured vascular endothelial cells. Toxicology, 117, 193-198.

Hirooka, T., Fujiwara, Y., Yamamoto, C., Yasutake, A. and Kaji, T. (2007): Methylmercury retards the repair of wounded monolayer of human brain microvascular endothelial cells by inhibiting their proliferation without nonspecific cell damage. J. Health Sci., 53, 450-456.

Iencean, S.M. (2003): Brain edema- a new classification. Med. Hypotheses, 61, 106-109.

Kaji, T., Fujiwara, Y., Hoshino, M., Yamamoto, C., Sakamoto, M. and Kozuka, H. (1995): Inhibitory effect of lead on the proliferation of cultured vascular endothelial cells. Toxicology, 95, 8792.

Knox, S., Merry, C., Stringer, S., Melrose, J. and Whitelock, J. (2002): Not all perlecans are created equal: interactions with fibroblast growth factor (FGF) 2 and FGF receptors. J. Biol. Chem., 277, 14657-14665.

Marmarou, A. (2004): The pathophysiology of brain edema and elevated intracranial pressure. Cleve. Clin. J. Med., 71 Suppl 1, 6-8.

Rifkin, D.B. and Moscatelli, D. (1989): Recent developments in the cell biology of basic fibroblast growth factor. J. Cell Biol., 109, 1-6.

Saksela, O., Moscatelli, D., Sommer, A. and Rifkin, D.B. (1988): Endothelial cell-derived heparan sulfate binds basic fibroblast growth factor and protects it from proteolytic degradation. J. Cell Biol., 107, 743-751.

Sanfeliu, C., Sebastià, J., Cristòfol, R. and Rodríguez-Farré, E. (2003): Neurotoxicity of organomercurial compounds. Neurotox. Res., 5, 283-306.

Sato, Y. and Rifkin, D.B. (1988): Autocrine activities of basic fibroblast growth factor: regulation of endothelial cell movement, plasminogen activator synthesis, and DNA synthesis. J. Cell Biol., 107, 1199-1205.

Yamamoto, C., Deng, X., Fujiwara, Y. and Kaji, T. (2005): Proteoglycans predominantly synthesized by human brain microvascular endothelial cells in culture are perlecan and biglycan. J. Health Sci., 51, 576-583. 
The FGF-2 system and the vascular toxicity of methylmercury

Yayon, A., Klagsbrun, M., Esko, J.D., Leder, P. and Ornitz, D.M. (1991): Cell surface, heparin-like molecules are required for binding of basic fibroblast growth factor to its high affinity receptor. Cell, 64, 841-848.
Zheng, W., Aschner, M. and Ghersi-Egea, J.F. (2003): Brain barrier systems: a new frontier in metal neurotoxicological research. Toxicol. Appl. Pharmacol., 192, 1-11. 
